\title{
Long-term outcome of mitomycin C-augmented needle revision of trabeculectomy blebs for late trabeculectomy failure
}

\author{
S. $\operatorname{Lin}^{1,2} \cdot$ D. Byles ${ }^{2} \cdot$ M. Smith $\mathbb{1}^{2}$
}

Received: 15 May 2018 / Revised: 19 July 2018 / Accepted: 4 August 2018 / Published online: 29 August 2018

(C) The Royal College of Ophthalmologists 2018

\begin{abstract}
Objectives To describe the 3-year outcomes of mitomycin C (MMC)-augmented bleb needling procedures following late trabeculectomy failure.

Methods A retrospective, observational chart review of 44 eyes that underwent MMC-augmented bleb needling following late trabeculectomy failure. The primary outcome measure was IOP at 3 years post-operatively. Secondary outcome measures were the number of topical glaucoma medications and success rate at 3 years. Success was defined on the basis of IOP ( $\leq 21 \mathrm{mmHg}$ and $\geq 5 \mathrm{mmHg}$ with a reduction of $\geq 20 \%$ from pre-operative baseline), need for ocular hypotensive medications, and need for further laser or surgical intervention.

Results Mean IOP was reduced from $19.8 \mathrm{mmHg}$ preoperatively to $13.9 \mathrm{mmHg}$ (29.5\% reduction) at 3 years. By the 3 -year time point, 26 eyes $(59.1 \%$ ) had met one or more of the criteria for failure. A lower immediate post-operative IOP was found to be associated with greater likelihood of success.

Conclusions Bleb needling augmented with MMC can achieve a significant reduction in IOP in eyes with late trabeculectomy failure. By 3 years however, 59\% of eyes were classified as failures, with half requiring further glaucoma surgery or laser. Patients undergoing this procedure should therefore be warned of the likelihood of requiring further intervention, and a repeat antimetabolite-augmented trabeculectomy or glaucoma tube shunt surgery in the first instance can be considered instead.
\end{abstract}

\section{Introduction}

Trabeculectomy surgery is the standard surgical treatment of choice for many glaucoma patients, where maximal tolerated medical therapy has failed to halt progression of glaucomatous optic neuropathy [1]. Although the procedure has a reasonably high success rate [2], a proportion of procedures do fail, and this can occur in the early postoperative period, or, in some cases, after a number of years. Intrableb scarring and fibrosis in the subconjunctival space is the most common cause of trabeculectomy failure, both in

M. Smith

s.lin@exeter.ac.uk

1 RILD Wellcome Wolfson Centre, University of Exeter Medical School, Royal Devon \& Exeter NHS Foundation Trust, Exeter, UK

2 West of England Eye Unit, Royal Devon \& Exeter NHS Foundation Trust, Exeter, UK the early and late post-operative periods, and results in the development of a failed, non-filtering bleb with an associated rise in intraocular pressure (IOP) [3]. Another common cause of a poorly draining trabeculectomy in the early post-operative period is encapsulation of the filtering bleb, also known as a Tenon's cyst. This usually occurs in within the first 6 months following surgery, and is caused by the formation of adhesions between the episcleral tissues and Tenon's capsule, resulting in a localised dome-shaped aqueous cyst [4].

Early bleb encapsulation can respond well to conservative management with IOP-lowering therapy, topical corticosteroids and digital ocular massage [4-9]. Late failing or failed trabeculectomy blebs, however, usually require further surgical intervention to restore aqueous flow. Transconjunctival needle revision, commonly referred to as "bleb needling", is a relatively simple technique to rescue bleb function with various surgical techniques described, all with a similar aim of dissecting the scar tissue adherent between the conjunctiva and the sclera [10-15]. This reopens the filtering site and restores the flow of aqueous 
through the pre-existing sclerectomy into the subconjunctival space, resulting in a lowering of IOP. The bleb needling procedure can be further augmented with the adjunctive use of antifibrotics such as 5fluorouracil (5-FU) and mitomycin C (MMC) in order to prevent post-needling fibrosis and improve success rates $[5,11,16]$.

The goal of this study was to determine the long-term outcomes and success rates of MMC-augmented bleb needling procedures following late trabeculectomy failure, and to identify factors associated with success or failure of the procedure.

\section{Methods}

A retrospective observational study was performed by chart review of all MMC-augmented bleb needling procedures following trabeculectomy performed at the West of England Eye Unit, Royal Devon \& Exeter Hospital between November 2008 and December 2013. As the study was a retrospective case note review, the local ethics committee confirmed that formal ethics approval was not required. The study adhered to the tenets of the Declaration of Helsinki. Patients were identified from electronic surgical theatre records and cross referenced against surgical logbooks maintained by the individual operating consultants. In order to exclude patients with selflimiting Tenon's cysts, patients who underwent bleb needing within 6 months of trabeculectomy surgery were excluded. Only patients with a minimum follow-up period of 12 months were included in the study analysis.

Ophthalmic history details were obtained from patient clinical records, with all previous ocular surgery and laser therapy documented. Pre-operative and postoperative topical ocular hypotensive therapies and IOPs measured by applanation tonometry were also obtained from patient clinical records, as well as all complications considered to be a direct consequence of the bleb needling procedure.

Where bilateral bleb needling procedures were performed in the same patient, each eye was analysed separately with both eyes included in the analysis. Only the first MMC-augmented bleb needling procedure performed in each eye was included in the study analysis, with the need for further bleb needling procedures considered a surgical failure.

\section{Technique of bleb needling procedure}

All bleb needling procedures were performed in the operating theatre. Under topical or sub-Tenon's anaesthesia, a superior corneal traction suture was placed and $0.15-0.2 \mathrm{ml}$ of $0.02 \% \mathrm{MMC}$ was injected into the superior subconjuctival space. A cellulose spear swab was used to disperse the MMC subconjunctivally in the area of the intended bleb. 5 min after injection the subconjunctival scar tissue was dissected using a 30-gauge needle or microvitreoretinal blade, depending on surgeon preference. If there was no drainage through the scleral flap, the flap was lifted, and the anterior chamber entered. Post-operatively, all glaucoma drops were stopped, and two hourly topical steroids commenced and continued for at least 2 months. The dosage of topical steroids was then tapered as clinically indicated.

\section{Outcome measures}

The primary outcome measure was IOP reduction at 3 years post-operatively. Secondary outcome measures were the number of topical glaucoma medications and the probability of success at 3 years post-operatively.

If the patient underwent further laser or surgery for glaucoma in the same eye, data were censored at the date of the additional procedure. Patients who had died or who were lost to follow-up by 24 or 36 months after the bleb needling procedure were omitted from the analysis of IOP and number of topical glaucoma medications at these time points.

Success was defined on the basis of IOP and need for further surgery. The procedure was considered a complete success if the IOP was $\leq 21 \mathrm{mmHg}$ and $\geq 5 \mathrm{mmHg}$ with an IOP reduction of $\geq 20 \%$ from pre-operative baseline without the need for any ocular hypotensive medications or need for further glaucoma laser or surgical intervention. The definition of a qualified success was identical, but with the aid of ocular hypotensive medications.

Potential risk factors for failure considered were age, gender, laterality, glaucoma diagnosis, lens status, previous glaucoma laser treatment (including argon laser trabeculoplasty, selective laser trabeculoplasty, Nd:YAG laser peripheral iridotomy and endoscopic cyclophotocoagulation), pre-needling IOP, immediate post-needling IOP, prior nonMMC augmented bleb needlings (with or without 5-FU injections), and interval between index operation and bleb needling procedure.

\section{Statistical analyses}

Tests of normality were applied to continuous data using the Shapiro-Wilk test. A two-tailed Student's t-test or a Wilcoxon signed-rank test was performed on paired parametric or non-parametetric data as appropriate to compare IOP and use of topical ocular hypotensive agents prior to and after bleb revision surgery. 
Kaplan-Meier plots were constructed to estimate the probability of survival over time without treatment failure after bleb needling, with censoring applied to patients who had died or who were lost to follow up. Binomial logistic regression analysis was used to assess the association between each study factor and IOP "survival" over time after bleb needling.

Statistical analyses were performed using an established software package (R Core Team 2015; R Foundation for Statistical Computing, Vienna, Austria) [17].

\section{Results}

In total, 47 eyes of 45 patients underwent bleb needling with adjunctive MMC following index filtration surgery during the study period. Follow-up data of at least 12 months were unavailable for three patients (all died within this time period), and these patients were excluded from further analyses. Furthermore, 44 eyes of 42 patients were therefore included in the study.

Patient demographics are summarised in Table 1. The mean age was 73.7 years (range $46.9-88.0$ years). Bleb needling procedures were performed on blebs related to 38 trabeculectomies (of which five were repeat trabeculectomies) and four trabeculectomies combined with phacoemulsification and intraocular lens implantation; the type of index procedure in one patient was unknown. Furthermore, 32 of these index procedures had been augmented with adjunctive antifibrotic agents (Table 1).

The mean interval between the index filtration surgery and first MMC-augmented bleb needling procedure was 76.4 months (range 6.8 months-23.8 years). The mean follow-up period following the first (or only) MMCaugmented bleb needling procedure until either the end of the study period or if the patient was lost to follow-up was 58.7 months (range 21.5 months- 8.5 years).

Complications after MMC-augmented bleb needling were observed in nine eyes (20\%). These included two eyes $(5 \%)$ with transient hyphema, one eye $(2 \%)$ with a transient immediate post-needling IOP spike and four eyes $(9 \%)$ with early post-operative hypotony and choroidal detachments. In all these cases the complication resolved spontaneously with no long-term adverse outcome. One eye $(2 \%)$ experienced a sustained post procedure IOP spike that necessitated further glaucoma tube shunt surgery, and one eye $(2 \%)$ developed a late bleb leak with hypotonous maculopathy 3 years following the bleb needling procedure that required further bleb revision surgery.

Pre-needling and post-needling IOP and number of topical ocular hypotensive medications required at 12, 24 and 36 months following the bleb needling procedure are
Table 1 Patient demographics and pre-operative characteristics

\begin{tabular}{|c|c|}
\hline Total number of patients & 42 \\
\hline \multirow[t]{2}{*}{ Total number of eyes } & \\
\hline & Number of eyes \\
\hline Gender & $\begin{array}{l}17(39 \%) \text { male } \\
27(61 \%) \text { female }\end{array}$ \\
\hline Laterality & $\begin{array}{l}15(34 \%) \text { right eye } \\
29(66 \%) \text { left eye }\end{array}$ \\
\hline $\begin{array}{l}\text { Lens status at time of first } \\
\text { MMC needling }\end{array}$ & $\begin{array}{l}28(64 \%) \text { pseudophakic } \\
16(36 \%) \text { phakic }\end{array}$ \\
\hline Diagnosis & $\begin{array}{l}29(66 \%) \text { primary open angle/norma } \\
\text { tension glaucoma } \\
7(16 \%) \text { chronic angle-closure } \\
\text { glaucoma } \\
5(11 \%) \text { pseudoexfoliation glaucoma } \\
2(5 \%) \text { uveitic glaucoma } \\
1(2 \%) \text { pigmentary glaucoma }\end{array}$ \\
\hline $\begin{array}{l}\text { Prior glaucoma laser } \\
\text { therapy }\end{array}$ & $\begin{array}{l}26(59 \%) \text { no prior laser therapy } \\
7(16 \%) \text { laser iridotomy } \\
4(9 \%) \text { ALT } \\
4(9 \%) \text { SLT } \\
3(7 \%) \text { had }>1 \text { prior laser } \\
\text { - } 1 \text { ALT + laser iridotomy } \\
\cdot 1 \text { ALT + cyclodiode + SLT } \\
\text { - } 1 \text { ALT + ECP }\end{array}$ \\
\hline $\begin{array}{l}\text { Number of prior bleb } \\
\text { needlings }+/-5 \text {-FU }\end{array}$ & $\begin{array}{l}34(77 \%) \text { No prior needling } \\
5(11 \%) 1 \text { prior needling } \\
1(2 \%) 2 \text { prior needlings } \\
2(5 \%) 3 \text { prior needlings } \\
1(2 \%) 4 \text { prior needlings } \\
1(2 \%) 6 \text { prior needlings }\end{array}$ \\
\hline Index filtration surgery & $\begin{array}{l}34(77 \%) \text { trabeculectomy } \\
\cdot 10 \text { standard } \\
\text { - } 3 \text { with perioperative 5-FU } \\
\text { - } 20 \text { with perioperative MMC } \\
\text { - } 1 \text { adjunct (if any) unknown } \\
5(11 \%) \text { repeat trabeculectomy } \\
\text { - } 1 \text { with perioperative } 5-F U \\
\text { - } 4 \text { with perioperative MMC } \\
4(9 \%) \text { phaco-trabeculectomy } \\
\text { - } 4 \text { with perioperative MMC } \\
1 \text { index surgery unknown }\end{array}$ \\
\hline
\end{tabular}

$S L T$ selective laser trabeculoplasty, $A L T$ argon laser trabeculoplasty, $E C P$ endoscopic cyclophotocoagulation, 5-FU 5-fluorouracil

${ }^{\text {a}}$ Phacoemulsification cataract surgery with intraocular lens implantation combined with trabeculectomy

summarised in Table 2. Mean IOP dropped from 19.8 $\mathrm{mmHg}$ preoperatively to $14.1 \mathrm{mmHg}$ (a drop of $28.9 \%$ ), $14.7 \mathrm{mmHg}$ (a drop of $25.8 \%$ ) and $13.9 \mathrm{mmHg}$ (a drop of $29.5 \%)$ at 12,24 and 36 months post-operatively; this reduction was statistically significant at all time points. There was also a reduction in number of topical IOPlowering therapies required from a mean of 1.7 preoperatively down to a mean of 1.2, 1.4 and 1.2 medications at 12,24 and 36 months post-operatively; this reduction was not statistically significant at all time points. 


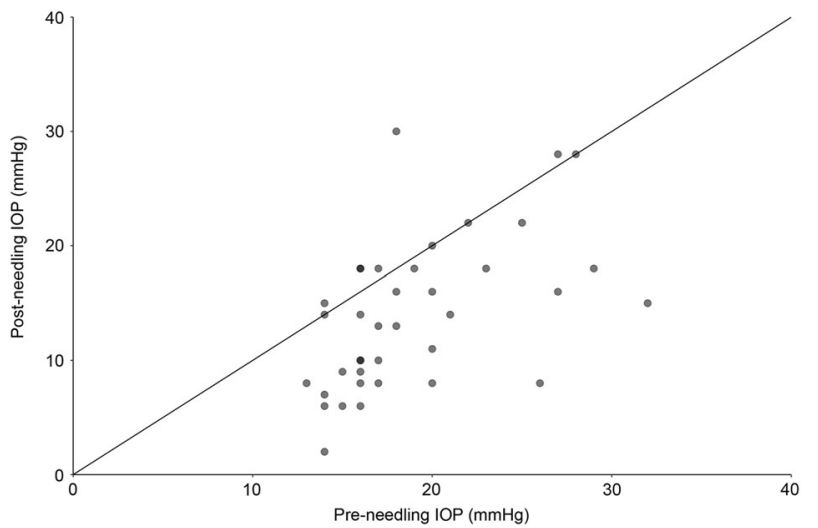

Fig. 1 A comparison of pre-operative and post-operative intraocular pressure (IOP) after bleb needling with adjunctive mitomycin $\mathrm{C}$, with a diagonal reference $(x=y)$ line

The change in IOP at 3 years post-operatively is illustrated in Fig. 1; most plotted points, each representing a treated eye, lie below the diagonal reference $(x=y)$ line, indicating a lower post-operative IOP for the majority of patients compared to the pre-operative baseline.

By the 3-year time point following bleb needling surgery, complete success was achieved in 12 eyes (27.3\%), and qualified success was achieved in a further four eyes $(9.1 \%)$; two eyes $(4.5 \%)$ were lost to follow up. Failure on the basis of IOP occurred in 22 eyes (50\%) following bleb needling surgery; 21 eyes (47.7\%) failed to achieve an adequate IOP reduction and post-operative hypotony occurred in one eye (2.3\%) following late bleb leakage. Furthermore, 22 eyes $(50.0 \%)$ underwent further glaucoma laser or surgical intervention, with a repeat bleb needling being the most common procedure performed. Of these, further bleb needling was performed in four eyes (9.1\%) despite an adequate IOP reduction according to defined success criteria on the basis of clinical indication. In three eyes this was due to subjective and objective visual field deterioration, and in one eye achieving qualified success this was due to an intolerance to topical ocular hypotensive medications. Reasons for failure and details of any further procedures performed are given in Table 3.

Kaplan-Meier survival analysis was used to assess the probability of achieving a complete or qualified success following MMC-augmented bleb needle revision (Fig. 2). At 3 years post-operatively, the probability of complete success was $31.2 \%$, and the probability of overall success (complete and qualified success) was $40.3 \%$.

A univariate binomial logistic regression analysis was used to assess potential risk factors for failure at the 3year time point following bleb needle revision. Risk factors assessed included age, gender, laterality, type of glaucoma (primary open angle glaucoma/normal tension 
glaucoma or other glaucoma diagnosis), lens status (phakic or pseudophakic), whether prior glaucoma laser or bleb needling performed (with or without 5-FU augmentation), pre-needling IOP, immediate post-needling IOP, and length of interval between index glaucoma surgery and first MMC-augmented bleb needling. Only one of the tested covariates, immediate post-needling IOP, was significantly associated with the outcome of the needling procedure $(p=0.039)$, with eyes that had undergone a successful needling procedure having a lower immediate post-needling IOP (mean $5.94 \mathrm{mmHg}$ ) compared to eyes in which needling was considered to have failed (mean $14.23 \mathrm{mmHg}$ ).

Table 3 Reasons for failure of bleb needling procedure

\begin{tabular}{ll}
\hline & $\begin{array}{l}\text { Number of eyes }(\% \\
\text { of total })\end{array}$ \\
\hline $\begin{array}{l}\text { Total number of bleb needling procedures } \\
\text { which failed }\end{array}$ & $26(59.1 \%)$ \\
$\begin{array}{l}\text { Reasons for failure: } \\
\text { IOP }>21 \mathrm{mmHg} \text {, or }<5 \mathrm{mmHg}, \text { or reduction }\end{array}$ & $22(50.0 \%)$ \\
of $<20 \%$ from pre-operative baseline & $22(50.0 \%)$ \\
Further laser or surgery performed (all): & $13(29.5 \%)$ \\
Repeat bleb needling & $5(11.4 \%)$ \\
Ahmed valve & $2(4.5 \%)$ \\
ECP & $1(2.3 \%)$ \\
Cyclodiode & $1(2.3 \%)$ \\
Bleb revision surgery &
\end{tabular}

$E C P$ endoscopic cyclophotocoagulation

\section{Discussion}

Bleb needle revision is a relatively simple and wellestablished procedure used to rescue bleb function in a failed or failing trabeculectomy. The success rate of the procedure can be further improved by the intraoperative use of adjunctive antifibrotics, which inhibit fibroblast activity and decrease post-needling fibrosis [18]. The first 5-FU augmented bleb needle revision was described in 1990, with MMC-augmented bleb needling procedures later described in 1996 [15, 19]. Since then, numerous studies have reported on the outcomes of MMC-augmented bleb needling procedures in restoring aqueous flow and improving IOP control following trabeculectomy bleb failure, with success rates ranging from 25 to $92 \%$ [11-13, 15, 20-28]. It is difficult to make comparisons across studies, however, due to the different demographics, sample sizes, needling techniques, MMC concentrations, duration of follow-up and definitions of success used.

In this series we found a mean post-needling IOP of 13.9 $\mathrm{mmHg}$ compared to a pre-needling IOP of $19.8 \mathrm{mmHg}$, representing an IOP reduction of $29.5 \%$. The 3 -year success rate was $31.1 \%$ without medications and $40.1 \%$ with or without medications. This success rate is lower than many previous reports of this procedure, and we feel that there may be several reasons for this.

Most studies describing the success rate of bleb needle revisions have combined eyes with both early and late trabeculectomy failure, and therefore include a proportion of patients with early bleb encapsulation, which may well have resolved without the need for any surgical intervention. As success has been correlated with needling procedures
Fig. 2 Kaplan-Meier survival analysis after bleb needling with adjunctive mitomycin $\mathrm{C}$. Complete success: IOP $\leq 21$ $\mathrm{mmHg}$ and $\geq 5 \mathrm{mmHg}$ with an IOP reduction of $\geq 20 \%$ from pre-operative baseline without the need for any further glaucoma laser or surgical intervention or ocular hypotensive medications. Complete \& Qualified success: IOP $\leq 21 \mathrm{mmHg}$ and $\geq 5 \mathrm{mmHg}$ with an IOP reduction of $\geq 20 \%$ from pre-operative baseline without the need for any further glaucoma laser or surgical intervention, with or without the need for ocular hypotensive medications. The number of eyes "surviving" at each time point is indicated in the risk table

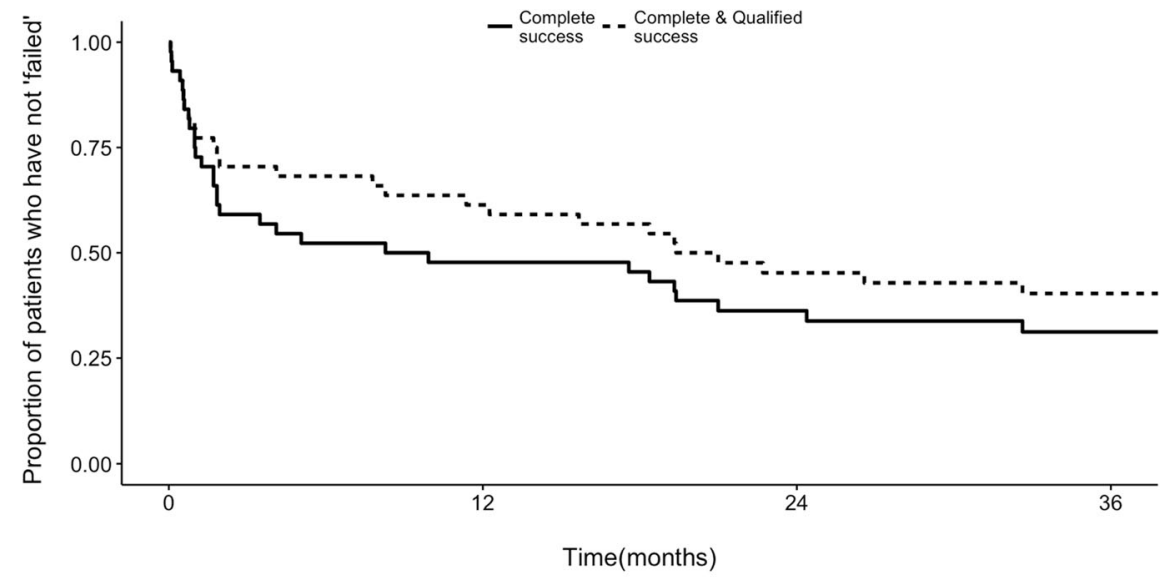

\begin{tabular}{|c|c|c|c|c|}
\hline \multicolumn{5}{|c|}{ Number at risk } \\
\hline $\begin{array}{r}\text { Complete } \\
\text { success }\end{array}$ & 44 & 21 & 15 & 12 \\
\hline $\begin{array}{r}\text { Complete \& Qualified } \\
\text { success }\end{array}$ & 44 & 27 & 19 & 16 \\
\hline & 0 & 12 & 24 & 36 \\
\hline
\end{tabular}


performed within 4 months of the glaucoma filtration surgery [12], this may account for the higher success rates reported in studies combining both early and late bleb needling. This is further supported by the findings from one study investigating bleb needling that was performed within 2-8 weeks of an unsuccessful glaucoma filtration surgery, which reported good success rates of $75 \%$ at 12 months following a single needle revision augmented with MMC.

Studies assessing the long-term efficacy of MMCaugmented bleb needling in the late post-operative period are limited. Maestrini et al. [24] studied 125 eyes that underwent needling procedures with adjunctive MMC between 6 months to 30 years following the last failed trabeculectomy and reported a success rate of $76 \%$ at the last follow-up. This figure represented the final success rate after one or more needling procedures, and included eyes that had undergone multiple needling procedures. When only the first needling procedure was considered, the success rate fell to $25.2 \%$ at 3 years. This was despite the study protocol allowing for further post-operative 5-FU or MMC injections following the needling procedure. Amini et al. [20] also included eyes which had undergone multiple needlings in their retrospective chart review of 27 patients who underwent needle revision with adjunctive MMC between 6 months to 5 years following the initial trabeculectomy surgery, and reported a success rate of $37 \%$ at 3 years.

In contrast to the above studies, we have used stricter criteria in defining success, where we have assessed outcomes following first MMC-augmented needle revision only. Failure of the primary needle revision procedure was said to occurred in eyes, which did not achieve an adequate IOP reduction or eyes that required any further glaucoma laser or surgical intervention, including subconjunctival injections of antifibrotics or further needle revisions.

It would therefore appear that the relatively low success rates in our series compared to previous studies relates to our series being restricted to the first MMC needling procedure (rather than allowing multiple procedures), only including late bleb failure (excluding early cases of selflimiting bleb encapsulation) and the 3-year follow-up period.

In this series, a lower immediate post-operative IOP was found to be associated with greater success, a finding that has been reported in several studies [16, 26, 29]. None of the other potential risk factors for failure studied were found to have a statistically significant effect on outcome.

The limitations of this study mainly relate to its retrospective nature. As a retrospective study we were not able to set the indications for bleb needling and our threshold for bleb needling may be different from other surgeons, and we appreciate this limits the applicability of the results to other surgeons. In addition, 4 (9.1\%) eyes underwent further procedures despite an adequate IOP reduction according to defined success criteria but, as a retrospective study, we were not able to set the threshold for further intervention, and this may influence the conclusions. Eight patients were also lost to follow-up prior to the 3-year follow-up time period, and were excluded from data analysis for the time points where the data were unavailable.

\section{Conclusions}

In conclusion, this study suggests that bleb needle revision with MMC can be successful in achieving a reduction in IOP in eyes with later trabeculectomy failure. By 3 years, however, only $36 \%$ met the success criteria, with half requiring further surgery or laser. Bleb needling is considered a relatively safe procedure with a low incidence of significant complications and rapid post-operative recovery. In view of the modest success of bleb needling in late trabeculectomy failure reported in this study however, we should counsel patients of the likelihood of requiring further intervention following a bleb needling procedure, and perhaps consider the option of a repeat antimetaboliteaugmented trabeculectomy or glaucoma tube shunt surgery in the first instance instead.

Although previous studies have reported higher success rates for this procedure we feel this may be related to the short follow-up intervals of these studies, combined with the inclusion of early cases of self-limiting bleb encapsulation and eyes which required multiple needling procedures.

\section{Summary}

\section{What was known before}

- Reports on the outcomes of needle revision for trabeculectomy bleb failure suggest the success rate is high, but studies vary in terms of inclusion criteria, definitions of success and the duration of follow up.

- There is limited evidence on the long-term success rate of a single needling procedure for late bleb failure

\section{What this study adds}

- Bleb needle revision with MMC can be successful in achieving a significant reduction in IOP in eyes with late trabeculectomy failure, but by 3 years $59 \%$ of cases did not meet the success criteria

- Although previous studies have reported higher success rates this may be related to a short duration of follow-up and the inclusion of both early cases of self-limiting bleb 
encapsulation and eyes, which required multiple procedures to achieve the final outcome.

\section{Compliance with ethical standards}

Conflict of interest The authors declare that they have no conflict of interest.

\section{References}

1. National Institute of Health and Care Excellence (2017). Glaucoma: diagnosis and management. NICE guideline (NG81). p. 13-15.

2. Landers J, Martin K, Sarkies N, Bourne R, Watson P. A twentyyear follow-up study of trabeculectomy: risk factors and outcomes. Ophthalmology. 2012;119:694-702.

3. Skuta GL, Parrish RK 2nd. Wound healing in glaucoma filtering surgery. Surv Ophthalmol. 1987;32:149-70.

4. Sherwood MB, Spaeth GL, Simmons ST, Nichols DA, Walsh AM, Steinmann WC, et al. Cysts of Tenon's capsule following filtration surgery. Medical management. Arch Ophthalmol. 1987; 105:1517-21.

5. Costa VP, Arcieri ES, Freitas TG. Long-term intraocular pressure control of eyes that developed encapsulated blebs following trabeculectomy. Eye (Lond). 2006;20:304-8.

6. Costa VP, Correa MM, Kara-Jose N. Needling versus medical treatment in encapsulated blebs. A randomized, prospective study. Ophthalmology. 1997;104:1215-20.

7. Scott DR, Quigley HA. Medical management of a high bleb phase after trabeculectomies. Ophthalmology. 1988;95:1169-73.

8. Shingleton BJ, Richter CU, Bellows AR, Hutchinson BT. Management of encapsulated filtration blebs. Ophthalmology. 1990; 97:63-68.

9. Suzuki R, Susanna R Jr. Early transconjunctival needling revision with 5-fluorouracil versus medical treatment in encapsulated blebs: a 12-month prospective study. Clin (Sao Paulo). 2013;68:1376-9.

10. Allen LE, Manuchehri K, Corridan PG. The treatment of encapsulated trabeculectomy blebs in an out-patient setting using a needling technique and subconjunctival 5-fluorouracil injection. Eye (Lond). 1998;12(Pt 1):119-23.

11. Anand N, Khan A. Long-term outcomes of needle revision of trabeculectomy blebs with mitomycin $\mathrm{C}$ and 5-fluorouracil: a comparative safety and efficacy report. J Glaucoma. 2009;18: 513-20.

12. Gutierrez-Ortiz C, Cabarga C, Teus MA. Prospective evaluation of preoperative factors associated with successful mitomycin $\mathrm{C}$ needling of failed filtration blebs. J Glaucoma. 2006;15:98-102.

13. Iwach AG, Delgado MF, Novack GD, Nguyen N, Wong PC. Transconjunctival mitomycin-C in needle revisions of failing filtering blebs. Ophthalmology. 2003;110:734-42.
14. Jacobs S, Gillis A, Van Malderen L, Zeyen T. Needling-revision of failed filtering blebs. Bull Soc Belge Ophtalmol. 2005;297: 59-64.

15. Shetty RK, Wartluft L, Moster MR. Slit-lamp needle revision of failed filtering blebs using high-dose mitomycin C. J Glaucoma. 2005;14:52-56.

16. Broadway DC, Bloom PA, Bunce C, Thiagarajan M, Khaw PT. Needle revision of failing and failed trabeculectomy blebs with adjunctive 5-fluorouracil: survival analysis. Ophthalmology. 2004;111:665-73.

17. R: A language and environment for statistical computing [computer program]. Vienna, Austria: R Foundation for Statistical Computing; 2015.

18. Lama PJ, Fechtner RD. Antifibrotics and wound healing in glaucoma surgery. Surv Ophthalmol. 2003;48:314-46.

19. Ewing RH, Stamper RL. Needle revision with and without 5fluorouracil for the treatment of failed filtering blebs. Am J Ophthalmol. 1990;110:254-9.

20. Amini H, Esmaili A, Zarei R, Amini N, Daneshvar R. Officebased slit-lamp needle revision with adjunctive mitomycin- $\mathrm{C}$ for late failed or encapsulated filtering blebs. Middle East Afr J Ophthalmol. 2012;19:216-21.

21. Ben-Simon GJ, Glovinsky Y. Needle revision of failed filtering blebs augmented with subconjunctival injection of mitomycin C. Ophthalmic Surg Lasers Imaging. 2003;34:94-99.

22. Groth S, Sponsel W. Effectiveness of needling revision with mitomycin-C for failing blebs. J Clin Exp Ophthalmol S. 2012;4:2.

23. Liu W, Wang J, Zhang M, Tao Y, Sun Y. Comparison of subconjunctival mitomycin $\mathrm{C}$ and 5-fluorouracil injection for needle revision of early failed trabeculectomy blebs. J Ophthalmol. 2016;2016:3762674.

24. Maestrini HA, Cronemberger S, Matoso HD, Reis JR, Merula RV, Filho AD, et al. Late needling of flat filtering blebs with adjunctive mitomycin C: efficacy and safety for the corneal endothelium. Ophthalmology. 2011;118:755-62.

25. Mahar P, Sami Memon A, Bhutto IA, Laghari DA Needle revision of failed drainage blebs with mitomycin-C. Pak J Ophthalmol. 2013; 29: 64-7.

26. Palejwala N, Ichhpujani P, Fakhraie G, Myers JS, Moster MR, Katz LJ. Single needle revision of failing filtration blebs: a retrospective comparative case series with 5-fluorouracil and mitomycin C. Eur J Ophthalmol. 2010;20:1026-34.

27. Pathak-Ray V, Choudhari N. Rescue of failing or failed trabeculectomy blebs with slit-lamp needling and adjunctive mitomycin C in Indian eyes. Indian J Ophthalmol. 2018;66: 71-76.

28. Tulidowicz-Bielak M, Kosior-Jarecka E, Zarnowski T. Revision of trabeculectomy filtering blebs with mitomycin C: long term results. Indian J Ophthalmol. 2016;64:822-8.

29. Shin DH, Kim YY, Ginde SY, Kim PH, Eliassi-Rad B, Khatana $\mathrm{AK}$, et al. Risk factors for failure of 5-fluorouracil needling revision for failed conjunctival filtration blebs. Am J Ophthalmol. 2001;132:875-80. 